\title{
LEARNING APPEARANCE PRIMITIVES OF IRIS IMAGES FOR ETHNIC CLASSIFICATION
}

\author{
Xianchao Qiu, Zhenan Sun, and Tieniu Tan \\ NLPR, Institute of Automation,Chinese Academy of Sciences \\ P.O. Box 2728, Beijing, P.R.China, 100080 \\ \{xcqiu, znsun, tnt $\} @$ nlpr.ia.ac.cn
}

\begin{abstract}
Iris pattern is commonly regarded as a kind of phenotypic feature without relation to genes. In our previous work, we argued that iris texture is race related, and its genetic information is illustrated in coarse scale texture features, rather than preserved in the minute local features of state-of-the-art iris recognition algorithms. In this paper, we propose a novel ethnic classification method based on learning appearance primitives of iris images. So we not only confirm that iris texture is race related, but also try to find out which kinds of iris visual primitives make iris images look different between Asian and non-Asian. In our scheme, we learned a small finite vocabulary of micro-structures, which are called IrisTextons, to represent visual primitives of iris images. Then we use Iris-Texton histogram to capture the difference between iris textures. Finally iris images are grouped into two race categories, Asian and non-Asian, by Support Vector Machine(SVM). Based on the proposed method, we get a higher correct classification rate(CCR) of $91.02 \%$ than our previous method on a database containing 2400 iris samples.
\end{abstract}

Index Terms - Iris recognition, SVM, image processing, ethnic classification.

\section{INTRODUCTION}

The iris of human eye is the annular part between the black pupil and the white sclera, in which texture is extremely rich. Some examples are shown in Fig. 1, which are from CASIABioSecure[1] iris database. Iris texture is random and unique to each subject[2, 3, 4], so iris recognition has become one of the most important biometric solutions for personal identification.

Since Daugman's[2] first iris recognition algorithm, there have been many schemes for iris representation and matching in literature. All these method regard iris texture as phenotypic feature[2, 3, 4]. That is to say, the iris texture is the result of the developmental process and is not dictated by genetics. Even the genetically identical irises, the right and left pair from any given person have different visual appearance.

However, through investigating a large number of iris images of different races, Asian and non-Asian, we found that these iris patterns have different visual appearance. Fig. 1 show some typical iris examples in CASIA-BioSecure[1] database, the first column is from Asian, the second column is from non-Asian. These images have very different visual appearance. For an iris image from Asian, the inner 1/2 annular part of iris region often provides more texture information than the outer $1 / 2$ annular part and the main patterns are spots and blocks. However for an iris image from non-Asian, the above two parts provide almost the same texture information and the main patterns are capillary like patterns. We have an intuitive assumption that the iris patterns are both a phenotypic feature and a genotypic feature. Iris texture of different race is consisted of different appearance primitives. Motivated by this assumption, we try to find out the differences of visual appearance in different ethnicity and do ethnic classification (also called race classification) based on iris texture.
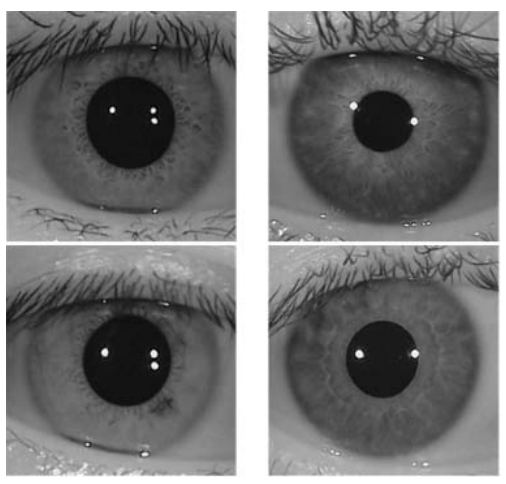

Fig. 1. Examples of iris images from CASIA-BioSecure iris databases. The first column is from Asian, the second column is from non-Asian.

In this paper, we propose a novel algorithm for automatic ethnic classification based on discriminative appearance primitives of iris images. The main purpose of this paper is to find appearance primitives of iris images and confirm the relationship between iris texture and genes. Firstly, compact and yet discriminative visual features, we call them Iris-Textons here, are automatically learned from a set of training images. Then the Iris-Texton histogram was used to represent the visual appearance of iris images. Finally, all images are classified into two ethnic categories, Asian and non-Asian, through a trained Support Vector Machine(SVM) 
classifier. This method is expected to be used for coarse classification in an iris recognition system. It will speed up iris matching on a very large scale iris database, which includes a lot of Asian and non-Asian users.

The remainder of this paper is organized as follows. Related works and background is presented in Section 2. The proposed method is discussed in Section 3. Experimental results are presented and discussed in Section 4 prior to conclusions in Section 5.

\section{RELATED WORKS AND BACKGROUND}

\subsection{Ethnic Classification}

Some attempts have been made to perform automatic ethnic classification based on images of human. One example is Gutta et al.[5] uses hybrid RBF/decision-trees and achieved an average accuracy rate of $92 \%$ for ethnic classification (consisting of four race groups: Asian, Oriental, Caucasian, and African). Recently, Viola et al.[6] used a variant of AdaBoost to classify face images into Asian and non-Asian. Their approach yields accuracy rate of 78\%. Lu and Jain[7] presented a Linear Discriminant Analysis (LDA) based scheme for two-class (Asian vs. non-Asian) ethnic classification from face images. Their reported accuracy is about $96 \%$. In our previous work[8], we do the same two-class ethnic classification based on global texture analysis of iris images and the accuracy is above $85 \%$.

\subsection{Texton Theory and Support Vector Machines}

Texton refers to fundamental micro-structure in generic natural images and thus constitute textures as the basic elements in early visual perception. The definition of texton is governed by a sound model of images in [9]. It is defined as a mini-template that represent certain appearance primitives in images. In fact, a small number of textons can be learned from training images as repeating appearance primitives. Then the texton histogram is used as a kind of global feature of an image. In practice, texton theory is widely used for image segmentation, texture recognition, texture synthesis, object detection and so on. We extend Texton theory to represent the visual appearance of iris images in this paper.

The SVM[10] is a technique for binary classification in the field of pattern recognition. This technique maps an input sample to a high-dimensional feature space and finds the optimal hyperplane that minimizes the recognition error for the training data using the linear and non-linear transformation function.

Let $n$ be the number of training samples. For the $i$ th sample $x_{i}$ with class label $c_{i} \in\{1,-1\}$, the SVM calculates

$$
f(x)=\sum_{i=1}^{n} \alpha_{i} c_{i} K\left(x, x_{i}\right)+b, \quad K\left(x, x_{i}\right)=\Phi(x) \cdot \Phi\left(x_{i}\right) .
$$

Coefficient $\alpha_{i}$ in Eq. 1 is non-zero when $x_{i}$ is a support vector that composes the hyperplane. Under all other condi- tions, it is zero. The kernel function $K\left(x, x_{i}\right)$ is easily computed by defining a inner product of the non-linear mapping function.

In this paper, a SVM is used for two-class (Asian and non-Asian) ethnic classification from iris images.

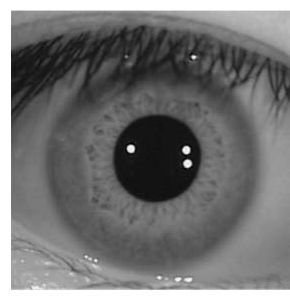

( a )

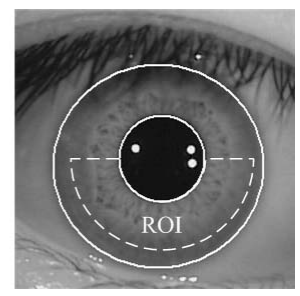

(b)

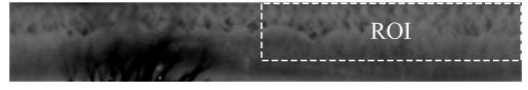

(c)

Fig. 2. Image preprocessing. (a) Original image. (b) Localized image. (c) Normalized and enhanced image.

\section{ETHNIC CLASSIFICATION WITH LEARNED VISUAL APPEARANCE}

\subsection{Image Preprocessing}

Image Preprocessing is very important in an iris recognition system. Fig. 2 illustrates the preprocessing steps involving localization, normalization and enhancement. More details can be found in [4]. To exclude the influence of eyelids and eyelashes, only the inner $3 / 4$ of the lower half of an iris region is used as the region of interest (ROI) for feature extraction, as shown in Fig. 2 (c). In our experiment, the size of $\mathrm{ROI}$ is $60 \times 256$.

\subsection{Learning the vocabulary of Iris-Textons}

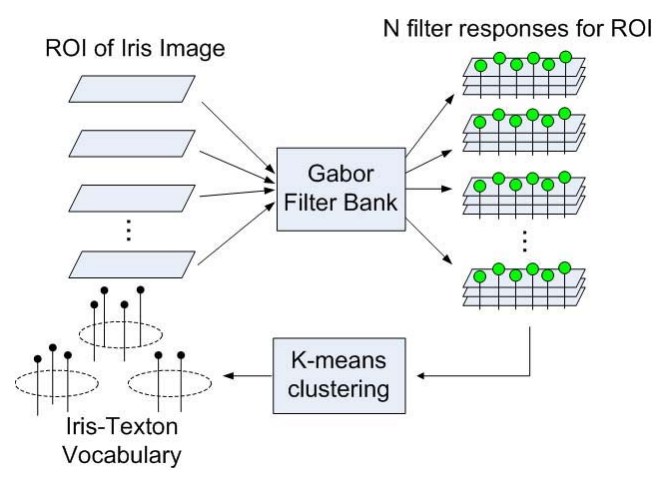

Fig. 3. The Iris-Texton vocabulary construction.

Firstly, we learn a small, finite vocabulary of appearance primitives in iris images, which are called Iris-Textons. A 
schematic diagram illustrating the steps of learning the universal Iris-Texton vocabulary is shown in Fig. 3.

The first step taken to learn vocabulary is filtering. As we know, the Gabor Filters have received considerable attention because the characteristics of certain cells in the visual cortex of some mammals can be approximated by these filters. In addition these filters have been shown to possess optimal localization properties in both spatial and frequency domain and thus are very suited for texture classification problems. Therefore we can characterize a texture by its responses to a set of orientation and spatial-frequency selective Gabor filters that cover the whole frequency space. Typically, an input image (ROI) $I(x, y)$ is convolved with a 2D Gabor filter (here we only use even Gabor filters) to obtain a Gabor filtered image $r(x, y)$.

$$
r(x, y)=\iint I\left(x_{1}, y_{1}\right) h\left(x-x_{1}, y-y_{1}\right) d x_{1} d y_{1}
$$

The computational models of 2D Gabor filters are:

$$
h(x, y)=g(x, y) \cdot \cos [2 \pi f(x \cos \theta+y \sin \theta)]
$$

where $g(x, y)$ is an isotropic Gaussian function given by

$$
g(x, y)=\frac{1}{2 \pi \sigma^{2}} \cdot \exp \left[-\frac{x^{2}+y^{2}}{2 \sigma^{2}}\right] .
$$

$f$ and $\theta$ are the spatial frequency and orientation respectively. In our experiments, there are a total of 40 even Gabor filters ( 8 orientations and 5 scales). Each pixel is then transformed to a $\mathrm{N}=40$ dimensional vector.

The second step is to cluster the filter response vectors into a small set of prototypes, Iris-Textons. These vectors are clustered using a vector quantization algorithm, in particular $\mathrm{K}$-means. K-means method try to find $\mathrm{K}$ centers such that after assigning each data vector to the nearest center, the sum of the squared distance from the centers are minimized. It is a greedy algorithm which iteratively performs the following two operations: (1) assign data vectors to the nearest of the $\mathrm{K}$ centers; (2) update each of the $\mathrm{K}$ centers to the mean of the data vectors assigned to it. These two steps are continued until the algorithm converges and a local minimum of the criterion is achieved. These centers are the Iris-Textons. The associated filter response vectors are called the appearance vectors. In this paper there are totally 64 Iris-Textons which are learned from 400 images from CASIA-BioSecure[1], 200 of them are random selected from Asian and the other 200 are from non-Asian.

\subsection{Representing Visual Appearance of Iris Image using Iris-Texton Histogram}

Once a vocabulary of Iris-Textons is learned, we can use IrisTexton histograms to represent the visual appearance of iris images. Each Iris-Texton is represented by its mean of the vectors in this cluster and it is one bin in the texton histogram. For a pixel of iris image, we get a $N=40$ dimensional vector by Gabor filtering and concatenation, and

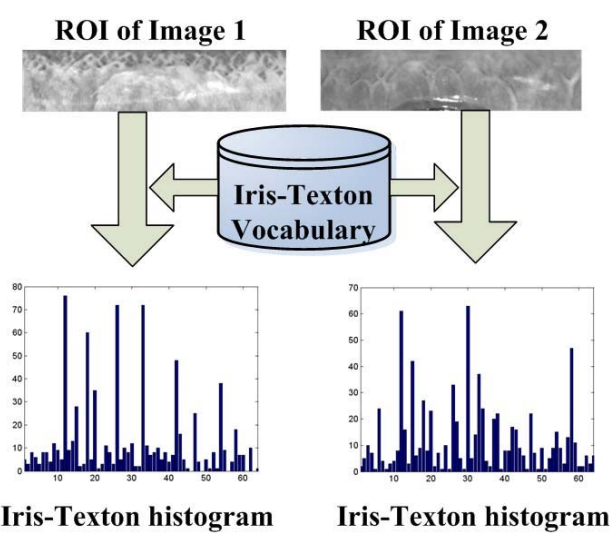

Fig. 4. The procedure of computing Iris-Texton Histograms.

assigned it to the bin which is the nearest texton. The IrisTexton histogram is a mapping of 40-dimensional vectors to 64 different Iris-Textons. We can see that the frequent variations of textons denote the richness of micro-textures in iris image. When proper filters are chosen, the Iris-Texton histogram is sufficient in characterizing visual appearance of iris images. The procedure of computing Iris-Texton Histograms is show in Fig. 4.

\subsection{Training SVM}

Before Iris-Texton histograms of the training set are applied to SVM, we need scaling each bin value to the range $[0,1]$. The kernel function chosen in our experiments was Radial Basis Function (RBF) with $\sigma=0.23$ and the value for the upper limit was $C=20$. We adopt cross-validation procedure to prevent the overfitting problem.

\section{EXPERIMENTAL RESULTS}

\subsection{Image database}

The experiment was done on the CASIA-BioSecure iris database, which includes 2400 images from 120 different eyes of 60 subjects. All images are captured by hand-held optical sensor. There are 20 images for each eye. Half of the images are captured in China from Asian, and the others are captured in France from non-Asian. Then the images are separated into two sets: a training set of 1200 images (600 images randomly selected from the Asian and 600 images randomly selected from the non-Asian) and a testing set of 1200 images (the remaining images).

\subsection{Results and Discussions}

Statistical test is carried out to measure the accuracy of this algorithm. Correct Classification Rate (CCR) of the algorithm is examined.

The ethnic classification result was show in Table 1. Compared with our previous method, the proposed method gets 


\begin{tabular}{|c|c|c|c|}
\hline \multirow{2}{*}{ Method } & \multicolumn{3}{|c|}{ Correct Classification Rate(\%) } \\
\cline { 2 - 4 } & Training Set & Testing Set & Overall \\
\hline Global Texture Analysis[8] & 84.83 & 85.67 & 85.25 \\
\hline The proposed method & $\mathbf{9 3 . 7 3}$ & $\mathbf{8 8 . 3 1}$ & $\mathbf{9 1 . 0 2}$ \\
\hline
\end{tabular}

Table 1. Comparison of classification accuracy between global texture analysis and the proposed method

higher CCR of $91.02 \%$.

Why the performance of this method is so good? Can Iris-Textons, iris visual primitives, really represent the iris visual difference between Asian and non-Asian? We compute the probability distribution of Iris-Textons in Asian and non-Asian iris images respectively in the training set. The distribution of Asian is sorted in descending order and we rearrange Iris-Textons with new labels from 1 to 64, as show in Fig. 5 (a). The distribution of non-Asian with the rearranged Iris-Texton labels is then illustrated in Fig. 5 (b). We can see that the two distributions are totally different, so we confirm that iris images from different races have different appearance primitives, iris texture is race related. Some typical iris patches according to top 10 Iris-Textons of Asian and non-Asian are given in Fig. 5 (c) and (d) respectively. These patches look very different, so Iris-Textons indeed depict the visual difference between Asian and non-Asian iris images.

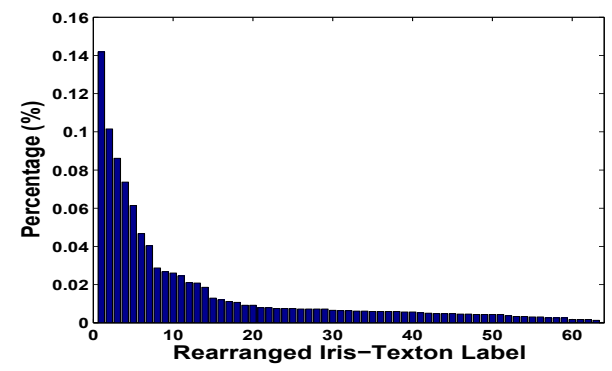

(a)Probability distribution of Iris-Textons in Asian

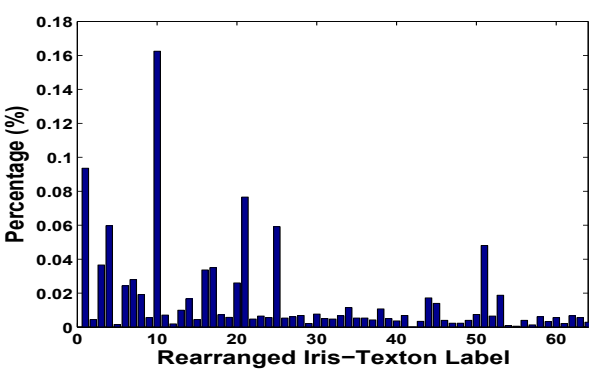

(b)Probability distribution of Iris-Textons in non-Asian

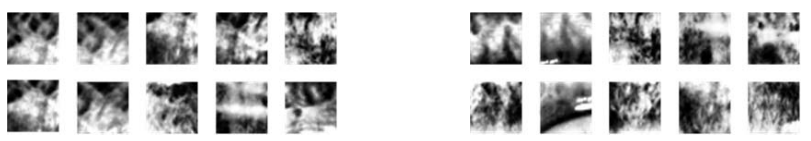

(c)Typical iris patches of Asian (d)Typical iris patches of non-Asian

Fig. 5. Iris-Texton distributions and typical iris patches of different Ethnic.

\section{CONCLUSION}

In this paper, we have presented a novel method for automatic ethnic classification based on discriminative visual appearance of iris images. The main contributions of this paper include: (1) A visual dictionary containing typical textons is learned from iris images. Each Iris-Texton characterizes a kind of frequently appeared local patches in iris images. So the global texture feature of an iris image are well presented by the distribution of its Iris-Textons. (2) We show that Asian and non-Asian iris images could be coarsely classified with histogram of Iris-Textons. The experimental results proves that global texture of iris image is genotypic.

In the future, we will extend the Iris-Texton concept, a powerful description of iris images, to iris recognition.

\section{ACKNOWLEDGEMENTS}

This work is funded by research grants from the National Basic Research Program (Grant No. 2004CB318110), the Natural Science Foundation of China (Grant No. 60335010, 60121302, 60275003, 60332010, 69825105, 60605008) and the Chinese Academy of Sciences.

\section{REFERENCES}

[1] CASIA-BioSecure Database, http://www.cbsria.ac.cn.

[2] J. Daugman, "High confidence visual recognition of persons by a test of statistical independence," IEEE TRANS. PAMI, vol. 15, no. 11, pp. 1148-1161, 1993.

[3] R.P. Wildes, "Iris recognition: An emerging biometric technology," Proceedings of the IEEE, vol. 85, no. 9, pp. 1348-1363, 1997.

[4] L. Ma, T. Tan, Y. Wang, and D. Zhang, "Personal identification based on iris texture analysis," IEEE TRANS. PAMI, vol. 25, no. 12, pp. 1519-1533, 2003.

[5] S. Gutta, H. Wechsler, and P. J. Phillips, "Gender and ethnic classification," in Proceedings of the 3rd FG, 1998, pp. 194-199.

[6] G. Shakhnarovich, P. A. Viola, and B. Moghaddam, "A unified learning framework for real time face detection and classification," in Proceedings of the 5th FG, 2002.

[7] X. Lu and A. K. Jain, "Ethnicity identification from face images," in Proc. SPIE Defense and Security Symposium, April 2004.

[8] X. Qiu, Z. Sun, and T. Tan, "Global texture analysis of iris images for ethnic classification," International Conference on Biometrics, pp. 411-418, 2006.

[9] S. Zhu, C. Guo, Y. Wang, and Z. Xu, "What are textons?," IJCV, vol. 62, no. 1-2, pp. 121-143, 2005.

[10] V. Vapnik, "Statistical learning theory," 1998. 